\title{
Neonatal Therapeutics: Considerations for Dosing
}

\author{
Kanecia O. Zimmerman, MD, MPH ${ }^{1,2}$ Daniel K. Benjamin Jr., , MD, PhD, MPH ${ }^{1,2}$ \\ Mara L. Becker, MD, MSCE ${ }^{1,2}$ \\ ${ }^{1}$ Duke Clinical Research Institute, Duke University School of Medicine, \\ Durham, North Carolina \\ 2 Department of Pediatrics, Duke University School of Medicine, \\ Durham, North Carolina \\ Address for correspondence Kanecia O. Zimmerman, MD, MPH, Duke \\ Clinical Research Institute, Box 3352, Durham, NC 27710 \\ (e-mail: kanecia.obie@dm.duke.edu).
}

Am J Perinatol 2019;36(suppl S2):S18-S21.
Abstract
Keywords
- neonate
- premature
- pharmacokinetics
- pharmacodynamic
- drugs
- absorption
- distribution
- metabolism
- elimination
- meropenem
- acyclovir
- physiology

Neonates are a uniquely vulnerable population, compromised by immature physiology and critical illness if born premature. Furthermore, neonates have frequent exposures to drugs that lack adequate data on safety, efficacy, and appropriate dosing in this population. Key physiologic differences between neonates and older children and adults affect drug absorption, distribution, metabolism, and elimination. Adequate understanding and consideration of these differences is essential to ensure optimal dosing of therapeutic agents in this vulnerable population. Moreover, direct study of neonates through appropriately designed pharmacokinetic and pharmacodynamic studies can ensure the development of safe and effective therapeutics in our youngest populations of patients.
Nearly half of neonatal therapeutics are used off-label, lacking adequate data on safety, efficacy, and appropriate dosing in this population. ${ }^{1}$ Dosing in neonates is particularly challenging; neonatal physiology differs substantially compared with older children and adults, which affects important components of drug disposition, including drug absorption, distribution, metabolism, and excretion. ${ }^{2}$ Adequate consideration of key differences is essential to ensure that therapeutic agents are safe and effective for this vulnerable population. Herein, we describe physiologic differences in neonatal drug absorption, distribution, metabolism, and elimination and provide examples that underscore the importance of these mechanisms in neonatal drug dosing.

\section{Absorption}

Drugs that are given by the oral route are absorbed through the mucosa of the gastrointestinal tract and may be influenced by numerous factors, including food or other drugs, luminal pH, gastric emptying time, and intestinal expression of transport proteins and drug-metabolizing enzymes. Compared with older children and adults, neonates have delayed gastric emptying time, decreased intestinal motility, and increased gastrointestinal wall permeability. ${ }^{2}$ Such physiology contributes to high variability in absorption. In addition, the alkaline $\mathrm{pH}$ in neonates results in increased bioavailability of acid-labile drugs (e.g., penicillins) and unexpectedly low bioavailability of weakly acidic drugs (e.g., phenobarbital and phenytoin). ${ }^{3}$

Transdermal application of drugs is increased in neonates due to a thinner stratum corneum, increased skin perfusion, and higher body surface area compared with body mass. ${ }^{4}$ Neonates also have higher proportions of total body and extracellular water and lower proportions of fat and muscle per body mass, resulting in greater absorption of intramuscular and percutaneous drugs that are water soluble. ${ }^{2}$ Greater absorption generally leads to higher plasma concentrations and increased risk for toxicity. Alternatively increased transdermal absorption can
Copyright (c) 2019 by Thieme Medical Publishers, Inc., 333 Seventh Avenue, New York, NY 10001, USA. Tel: +1(212) 584-4662 ISSN 0735-1631. 
be leveraged to minimize more invasive methods of administration. In 20 infants, $\leq 30$ weeks gestational age (GA) transdermal theophylline gel resulted in adequate blood levels for up to 70 hours. $^{5}$

\section{Distribution}

In addition to its effect on drug absorption, body composition markedly contributes to drug distribution. Water constitutes approximately $80 \%$ of total body mass in neonates and declines to the adult value of $60 \%$ within the first year of life, resulting in increased volume of distribution of water soluble drugs in neonates relative to adults, and lower drug concentrations in plasma. $^{2}$ In addition, lower protein concentrations and lower affinity for drug binding in neonates compared with older children and adults can result in greater drug effect at a lower dose. ${ }^{2}$ Furthermore, after the first week of life, bilirubin has increasing affinity for albumin; hyperbilirubinemia, a common finding in this population, can further limit drug-protein binding and enhance concentrations of unbound drug. ${ }^{6}$

Neonatal physiology has especially important implications for drug distribution to organs such as the brain. Neonates exhibit higher permeability of the blood-brain barrier and a higher ratio of cerebral to systemic blood flow compared with older children and adults. ${ }^{7}$ Combined with higher concentrations of unbound protein, these factors can result in higher central nervous system (CNS) concentrations for drugs with usually limited permeability, increased efficacy at a lower drug dose, and the possibility for more frequent or severe adverse events of CNS.

Acyclovir for proven or suspected neonatal herpes simplex virus (HSV) infection highlights the importance of physiologic consideration in neonatal dosing, particularly with regard to optimal distribution to target tissues-the skin, eyes, and mouth; the CNS; or throughout the body. To prevent HSVassociated death or severe neurodevelopmental impairment, administration of $20 \mathrm{mg} / \mathrm{kg} /$ dose is the standard of care for neonates $\leq 28$ days postnatal age (PNA), based on evidence of decreased mortality and improved neurodevelopmental outcomes compared with the prior dosing recommendations (10 $\mathrm{mg} / \mathrm{kg} /$ dose $)^{8}$ Various neonatal dosing guides suggest that high-dose therapy should be given two to three times daily based on GA or postmenstrual age (PMA).

Rapid physiologic changes across the pediatric age continuum support stratified dosing by age. Prematurity is associated with significantly lower albumin concentrations and greater volumes of distribution compared with that of term gestation. ${ }^{9}$ However, these infants may also experience hypotension, low cardiac output, and inadequate tissue perfusion due to sepsis or shock, which may counter normal physiologic processes and result in greater uncertainty regarding drug exposures.

Physicochemical properties of acyclovir also encourage age-stratified dosing. Acyclovir levels in the cerebrospinal fluid only reach 30 to $50 \%$ of corresponding plasma levels; therefore, dosing must achieve plasma levels of $\geq 3 \mathrm{mg} / \mathrm{L}$ to maintain target concentrations of $\geq 1 \mathrm{mg} / \mathrm{L}$ in the CNS. ${ }^{10}$ However, therapeutic dosing must account for the limited protein binding of acyclovir (22\%) and increased permeability of the blood-brain barrier to avoid supratherapeutic CNS concentrations that may increase the risk for neurotoxicity in premature infants. $^{11}$

Pursuant to these safety concerns and to achieving therapeutic concentrations for $>50 \%$ of the dosing interval, pharmacokinetic analysis of acyclovir in 28 infants 23 to 42 weeks GA and $<31$ days PNA resulted in the following recommended dosing regimens: $<30$ weeks PMA: $20 \mathrm{mg} / \mathrm{kg}$ every 12 hours; 30 to 36 weeks PMA: $20 \mathrm{mg} / \mathrm{kg}$ every 8 hours; and 36 to 41 weeks PMA: $20 \mathrm{mg} / \mathrm{kg}$ every 6 hours. $^{12}$ Consistent with these analyses, dosing based on PMA is now Food and Drug Administration (FDA) approved for incorporation into the acyclovir product label.

\section{Metabolism}

Neonatal drug metabolism is largely determined by the expression of drug-metabolizing enzymes that can impact both phase I and phase II reactions. Enzyme expression exhibits notable ontogeny, often low at birth and increasing over time to adult levels. ${ }^{2}$ For example, cytochrome P450 (CYP) $3 \mathrm{~A} 4$ levels are absent to low in fetal samples and increase to $50 \%$ of adult levels between 6 and 12 months PNA. ${ }^{13}$ However, some CYP levels decrease over time; significantly elevated CYP3A7 levels were identified in premature liver microsomes and decreased steadily through 6 months of age. ${ }^{13}$ Development can also affect phase II reactions such as acetaminophen glucuronidation and sulfation. ${ }^{14}$ In addition, lower protein binding in neonates may lead to higher hepatic metabolism. These processes can result in altered drug concentrations relative to older children and adults.

Recent data regarding sildenafil pharmacokinetics in term and preterm infants illustrate the relevance of understanding CYP enzyme ontogeny in this population. In term infants up to 7 days PNA, interpatient variability of sildenafil concentration was high. ${ }^{15}$ In preterm infants up to 209 days PNA, plasma concentrations were similar to those in term infants; and infants who received inducers of CYP3A4 and CYP2C9 as comedications had higher metabolite-to-parent ratios compared with nonreceipt of similar medications. ${ }^{16}$ Such evidence suggests that PNA, independent of GA, has a notable effect on CYP3A4 maturation; need for dose adjustment may be limited to soon after birth; and CYP3A4 activity is inducible even in the premature infant. Further, high interpatient variability and competing physiologic processes underscore the importance of direct study of pharmacokinetics, safety, and efficacy in neonates.

\section{Elimination}

Neonatal physiology also results in altered drug elimination pathways compared with older children and adults. Specifically, mechanisms of renal excretion, including glomerular filtration, active tubular secretion, and tubular reabsorption, increase with increasing GA, PNA, and body weight. ${ }^{17}$ Glomerular filtration rate (GFR) for body surface area is initially low in term neonates, increasing rapidly during the first 2 weeks of life, subsequently rising slowly to adult values by 
6 to 12 months PNA. ${ }^{17}$ In premature infants, incomplete nephrogenesis until 34 weeks' gestation limits initial rapid rises in GFR. ${ }^{17}$ Notably, conditions common in preterm neonates such as decreased cardiac output and receipt of nephrotoxic drugs can also result in lower GFR.

Elimination pathways of active tubular secretion and tubular reabsorption are similarly immature at birth, increasing the risk for prolonged and toxic drug exposures in neonates. Initially, elimination pathways are only 20 to $30 \%$ of that observed in adults, with active tubular secretion gradually reaching adult values by 7 to 12 months of life and tubular reabsorption maturing through adolescence. ${ }^{2}$ Meropenem provides a pertinent example of the importance of careful consideration of these pathways in neonatal drug dosing.

Meropenem, a $\beta$ lactam administered intravenously, has broad antimicrobial activity with stability against resistance mechanisms of most extended spectrum and AmpC chromosomal $\beta$-lactamases. ${ }^{18}$ Meropenem has low protein binding ( $2 \%$ in adults) and broad distribution and tissue penetration in the body. ${ }^{18}$ Consequently, this drug is used for treatment of meningitis, late-onset sepsis, and intraabdominal infections in neonates. ${ }^{19,20}$ Meropenem undergoes limited metabolism to an inactive metabolite, and $>70 \%$ of the drug is excreted unchanged in the urine, dependent on both glomerular filtration and active tubular secretion for clearance. ${ }^{18}$ As such, adjustment in dose is recommended for creatinine clearance $<50 \mathrm{~mL} / \mathrm{min}$, geriatric patients, and recently, neonates, including those who are premature. ${ }^{18}$

In 23 premature and 15 full-term neonates, meropenem administered at doses of 10,20 , or $40 \mathrm{mg} / \mathrm{kg}$ over 30 minutes resulted in substantially lower clearance among preterm (0.253-0.398 L/h) compared with term neonates (0.414$0.753 \mathrm{~L} / \mathrm{h}$ ) and a half-life up to 3.8 hours in preterm infants compared with 1 hour in adults. ${ }^{21}$ Based on findings among 200 premature and term infants $<91$ days old, FDA-recommended dosing considers both GA and PNA: GA $<32$ weeks, PNA $<2$ weeks: $20 \mathrm{mg} / \mathrm{kg}$ every 12 hours; GA $<32$ weeks, PNA $\geq 2$ weeks: $20 \mathrm{mg} / \mathrm{kg}$ every 8 hours; GA $\geq 32$ weeks, PNA $<2$ weeks: $20 \mathrm{mg} / \mathrm{kg}$ every 8 hours; and GA $\geq 32$ weeks, PNA $\geq 2$ weeks: $30 \mathrm{mg} / \mathrm{kg}$ every 8 hours. ${ }^{20}$

As demonstrated by the example of meropenem, neonatal physiology should also be considered when determining the method of administration. Due to meropenem's time-dependent killing, prolonged or continuous infusion has been recommended in adults. However, in a study comparing a standard 30-minute infusion of meropenem versus a 4-hour prolonged infusion in infants with very low birth weight, there was no difference in time of drug levels above minimum inhibitory concentration. ${ }^{22}$ The lack of difference could be explained by the lower clearance of meropenem in less mature neonates compared with those more mature. In addition, given evidence of CNS penetration of $>40 \%$ with inflamed meninges (evidenced by greater protein concentration), ${ }^{23}$ prolonged infusion strategies applied to more premature infants may increase the risk for neurotoxicity, including the development of seizures known to be associated with carbapenems such as meropenem. However, in the absence of CNS inflammation, penetration of the CNS may be as low as $8 \%{ }^{23}$

\section{Conclusion}

In this era of precision medicine, the goal is to get the right drug, to the right patient, at the right dose, at the right time to maximize drug efficacy and minimize drug-related toxicities. Hospitalized neonates represent a challenging subset of patients to treat accurately and effectively due to rapidly changing physiology. We have illustrated where thoughtfully planned pharmacokinetic studies in this unique subset of patients has resulted in drug dosing and labeling changes. Continued strategic pharmacokinetic and pharmacodynamic investigation in this vulnerable population will ensure that accurate and effective drug dosing occurs and optimal outcomes are achieved.

\section{Conflict of Interest}

K.O.Z. receives support from the National Institutes of Health (National Institute of Child Health and Human Development (K23 HD091398, HHSN275201000003I), the Duke Clinical and Translational Science Award (KL2TR001 115-03), and industry for neonatal and pediatric drug development, www.dcri.duke.edu/research/coi.jsp. D.K.B. Jr. receives support from the National Institutes of Health (award 2K24HD058735-10, National Institute of Child Health and Human Development (HHSN275201000003I), National Institute of Allergy and Infectious Diseases (HHSN272201500006I), ECHO Program (1U2COD02337502), and the National Center for Advancing Translational Sciences (1U24TR001608-03); he also receives research support from Cempra Pharmaceuticals (subaward to HHS 0100201300009C) and industry for neonatal and pediatric drug development, www.dcri.duke.edu/research/coi.jsp. M. L.B. receives support from the National Institutes of Health (National Institute of Child Health and Human Development [R01HD089928] and the National Center for Advancing Translational Sciences [1U24TR001608-03]); she also has a consulting agreement in place with Swedish Orphan Biovitrum for pharmacokinetic consultation for anakinra in the pediatric population.

\section{References}

1 Costa HTML, Costa TX, Martins RR, Oliveira AG. Use of off-label and unlicensed medicines in neonatal intensive care. PLoS One 2018;13(09):e0204427

2 Kearns GL, Abdel-Rahman SM, Alander SW, Blowey DL, Leeder JS, Kauffman RE. Developmental pharmacology-drug disposition, action, and therapy in infants and children. N Engl J Med 2003; 349(12):1157-1167

3 Huang NN, High RH. Comparison of serum levels following the administration of oral and parenteral preparations of penicillin to infants and children of various age groups. J Pediatr 1953;42(06): 657-658

4 Stamatas GN, Nikolovski J, Luedtke MA, Kollias N, Wiegand BC. Infant skin microstructure assessed in vivo differs from adult skin in organization and at the cellular level. Pediatr Dermatol 2010;27 (02):125-131

5 Evans NJ, Rutter N, Hadgraft J, Parr G. Percutaneous administration of theophylline in the preterm infant. J Pediatr 1985;107(02): 307-311 
6 Kapitulnik J, Blondheim SH, Kaufmann NA. Sephadex absorption of bilirubin from neonatal and adult serum. Clin Chem 1972;18 (01):43-47

7 Caley DW, Maxwell DS. Development of the blood vessels and extracellular spaces during postnatal maturation of rat cerebral cortex. J Comp Neurol 1970;138(01):31-47

8 Kimberlin DW, Lin CY, Jacobs RF, et al; National Institute of Allergy and Infectious Diseases Collaborative Antiviral Study Group. Safety and efficacy of high-dose intravenous acyclovir in the management of neonatal herpes simplex virus infections. Pediatrics 2001;108(02):230-238

9 Cartlidge PH, Rutter N. Serum albumin concentrations and oedema in the newborn. Arch Dis Child 1986;61(07):657-660

10 Rabalais GP, Nusinoff-Lehrman S, Arvin AM, Levin MJ. Antiviral susceptibilities of herpes simplex virus isolates from infants with recurrent mucocutaneous lesions after neonatal infection. Pediatr Infect Dis J 1989;8(04):221-223

11 Haefeli WE, Schoenenberger RA, Weiss P, Ritz RF. Acyclovirinduced neurotoxicity: concentration-side effect relationship in acyclovir overdose. Am J Med 1993;94(02):212-215

12 Sampson MR, Bloom BT, Lenfestey RW, et al; Best Pharmaceuticals for Children Act-Pediatric Trials Network. Population pharmacokinetics of intravenous acyclovir in preterm and term infants. Pediatr Infect Dis J 2014;33(01):42-49

13 Lacroix D, Sonnier M, Moncion A, Cheron G, Cresteil T. Expression of CYP3A in the human liver-evidence that the shift between CYP3A7 and CYP3A4 occurs immediately after birth. Eur J Biochem 1997;247(02):625-634

14 Miller RP, Roberts RJ, Fischer LJ. Acetaminophen elimination kinetics in neonates, children, and adults. Clin Pharmacol Ther 1976;19(03):284-294
15 Mukherjee A, Dombi T, Wittke B, Lalonde R. Population pharmacokinetics of sildenafil in term neonates: evidence of rapid maturation of metabolic clearance in the early postnatal period. Clin Pharmacol Ther 2009;85(01):56-63

16 Thakkar N, Gonzalez D, Cohen-Wolkowiez M, et al. An opportunistic study evaluating pharmacokinetics of sildenafil for the treatment of pulmonary hypertension in infants. J Perinatol 2016;36(09):744-747

17 Arant BS Jr. Developmental patterns of renal functional maturation compared in the human neonate. J Pediatr 1978;92(05): 705-712

18 United States National Library of Medicine. DailyMed. Available at: https://dailymed.nlm.nih.gov/. Accessed February 15, 2019

19 Lutsar I, Chazallon C, Trafojer U, et al. Meropenem vs standard of care for treatment of neonatal late onset sepsis (NeoMero1): a randomised controlled trial. bioRxiv 2018. Doi: 10.1101/456871

20 Smith PB, Cohen-Wolkowiez M, Castro LM, et al; Meropenem Study Team. Population pharmacokinetics of meropenem in plasma and cerebrospinal fluid of infants with suspected or complicated intra-abdominal infections. Pediatr Infect Dis J 2011;30(10):844-849

21 van den Anker JN, Pokorna P, Kinzig-Schippers M, et al. Meropenem pharmacokinetics in the newborn. Antimicrob Agents Chemother 2009;53(09):3871-3879

22 Padari H, Metsvaht T, Kõrgvee LT, et al. Short versus long infusion of meropenem in very-low-birth-weight neonates. Antimicrob Agents Chemother 2012;56(09):4760-4764

23 Germovsek E, Lutsar I, Kipper K, et al; NeoMero Consortium. Plasma and CSF pharmacokinetics of meropenem in neonates and young infants: results from the NeoMero studies. J Antimicrob Chemother 2018;73(07):1908-1916 